\title{
Resultados clínicos e imagenológicos en pacientes con rinosinusitis crónica odontogénica
}

\author{
Evaluation of clinical and imaging results in patients \\ with chronic odontogenic rhinosinusitis
}

\author{
Romina Novoa $\mathbf{R}^{1}$, Ilson Sepúlveda A $^{2}$, Juan Munzenmayer $\mathbf{B}^{3}$, Thomas Schmidt $\mathbf{P}^{1,2}$.
}

\begin{abstract}
RESUMEN
Introducción: La rinosinusitis crónica odontogénica (RSCO) es un proceso inflamatorio/infeccioso de la mucosa nasal y senos paranasales (SPN) de origen dental. Corresponde a una patología subdiagnosticada en la actualidad. El gold standard diagnóstico es la tomografía computarizada. Los tratamientos difieren, desde tratamiento médico aislado hasta médico-quirúrgico de SPN o combinados.

objetivo: Caracterizar los resultados clínicos e imagenológicos de pacientes con RSCO en función del tratamiento recibido, para establecer recomendaciones terapéuticas. Describir la frecuencia de subdiagnóstico de esta patología.

Material y método: Estudio descriptivo, retrospectivo. Se incluyeron pacientes con diagnóstico clínico e imagenológico de RSCO entre los años 2013-2017 en un centro de atención médico privado, a los que se realizó una tomografía computarizada cone beam de control. Fueron excluidos aquellos con rinosinusitis crónica, cirugía endoscópica funcional (CEF) previa, cáncer, embarazadas, rechazo a tomografía computarizada cone beam, ausencia de imágenes preoperatorias y menores de 18 años.

Resultados: De un total de 27 pacientes, en los operados de CEF ( $n=24 ; 89 \%)$ la resolución total de los síntomas se logró en 20/24 (83\%), resolución parcial 3/24 (12,5\%) y persistencia de los síntomas 1/24 (4\%).

Discusión: De los pacientes con RSCO 22/27 (81\%) no tenían reportado el foco dental en el informe radiológico a pesar de ser visible. Aquellos pacientes con sintomatología postratamiento, hubo factores identificados como la bilateralidad, mala dentadura general, falta de combinación de tratamiento dental y CEF.

Conclusiones: La CEF combinada con tratamiento dental concomitante, tiene un alto grado de éxito radiológico y clínico en esta patología. No fue posible demostrar si siempre es requerida la exodoncia con cierre de fístula oro-antral o si se pueden seleccionar pacientes para un tratamiento más conservador.
\end{abstract}

Palabras clave: Sinusitis odontogénica/sinusitis maxilar, tomografía computarizada cone beam, cirugía endoscópica funcional, exodoncia/cirugía oral.

\footnotetext{
1 Departamento Otorrinolaringología, Facultad de Medicina, Universidad de Concepción, Concepción, Chile.

2 Servicio de Otorrinolaringología y Cabeza y Cuello, Hospital Regional Guillermo Grant Benavente, Concepción, Chile.

3 Servicio Maxilofacial, Hospital Regional Guillermo Grant Benavente, Concepción, Chile.
}

Los autores declaran no tener conflictos de interés.

Recibido el 20 de septiembre, 2019. Aceptado el 12 de enero, 2020. 


\section{ABSTRACT}

Introduction: Chronic odontogenic rhinosinusitis (CORS) is an inflammatory/infectious process of the nasal mucosa and sinuses of dental origin. Actually, it corresponds to an underdiagnosed pathology. The gold standard diagnosis is computed tomography. The treatments differ, from isolated medical treatment to medical-surgical sinuses or combined.

Aim: To characterize the clinical and imaging results of patients with CORS according to the treatment received, to establish therapeutic recommendations. In addition to describing the frequency of subdiagnosis of this pathology.

Material and method: Descriptive, retrospective study. Patients with clinical and imaging diagnosis of CORS between 2013-2017 were included in a private medical care center, to whom a cone beam computed tomography control was performed. Those with chronic rhinosinusitis, previous functional endoscopic sinus surgery (FESS), cancer, pregnant women, rejection of cone beam computed tomography, absence of preoperative images and those under 18 years were excluded.

Results: Of all patients operated on FESS ( $n=24 ; 89 \%)$ the total resolution of the symptoms was 20/24 (83\%), partial resolution 3/24 (12.5\%) and persistence of the symptoms a $1 / 24(4 \%)$.

Discussion: 22/27 (81\%) of patients with CORS had not reported the dental focus in the radiological report despite being visible. Those patients with post-treatment symptoms had identified factors such as bilaterality, general bad teeth, lack of combination of dental treatment and FESS.

Conclusion: The FESS combined with concomitant dental treatment, has a high degree of radiological and clinical success in this pathology. It was not possible to demonstrate whether exodontia with closure of oroantral fistula is always required or if patients can be selected for a more conservative treatment.

Key words: Odontogenic sinusitis/maxillary sinusitis, cone beam computed tomography, endoscopic surgery, FESS, exodontics/oral surgery.

\section{INTRODUCCIÓN}

La rinosinusitis odontogénica (RSO) es un proceso inflamatorio/infeccioso de la mucosa nasal y cavidades paranasales de origen dental, pudiendo presentar un curso agudo o crónico ${ }^{1}$. Los reportes epidemiológicos de rinosinusitiscrónicaenel mundo, estiman una prevalencia de 6\%-16\% en Estados Unidos y de 7\%-27\% en Europa, correspondiendo $89 \%$ a origen rinógeno y aproximadamente $11 \%$ a origen odontogénico ${ }^{2,3}$. Se ha detectado en estudios imagenológicos de rinosinusitis que en aquellos casos con compromiso bilateral de las cavidades paranasales $6 \%$ corresponde a origen dental, en cambio cuando existe un compromiso unilateral, hasta $75 \%$ puede corresponder a causa odontogénica ${ }^{4,5}$. La etiología dental reúne varias causas, como procesos infecciosos periodontales, traumatismo dental y la iatrogenia, siendo la causa más frecuente esta última. La iatrogenia incluye la extracción dental incompleta, implantes dentales infectados, endodoncias, elevaciones de alveolo maxilar y cirugías ortognáticas con infección del material implantado o por secuestros óseos $s^{6,7}$.

La fisiopatología se explica a partir de una pieza dental y tejido periapical sano que se ven sometido a un proceso infeccioso desde el área periodontal, pulpa dental o trauma directo de la membrana schneideriana generando inflamación y/o disrupción de ella, con 0 sin la consiguiente formación de una fístula oro-antral (FOA). Esto provoca la inflamación, de distinta magnitud, de la mucosa adyacente del seno maxilar, con la posible extensión por contigüidad hasta el complejo osteomeatal (COM) y otros senos paranasales $(\mathrm{SPN})^{8}$. Se ha descrito además la formación de biofilms en esta zona de comunicación/inflamación, siendo ésta la posible explicación a la falla del tratamiento antibiótico ${ }^{9}$. 
Para hacer el diagnóstico se deben reunir los criterios de EPOS 2012, a los que se agregan síntomas y signos sugerentes de rinosinusitis crónica odontogénica (RSCO) como la unilateralidad de los síntomas, cacosmia, dolor a la presión o percusión de la pieza dental y antecedentes de procedimientos dentales adyacentes al seno maxilar ${ }^{1,10}$. Lo que se busca es identificar un proceso dental que pueda ser la causa de los síntomas ${ }^{11}$. Para ello se han utilizado diferentes métodos imagenológicos desde una radiografía dental hasta la tomografía computarizada cone beam. La radiografía dental es el examen radiológico más usado para detectar periodontitis apical, pero al ser una imagen en $2 \mathrm{D}$ se genera superposición de estructuras, con una alta tasa de falsos negativos. La radiografía panorámica permite ver la neumatización del seno, la relación diente-maxilar, pseudoquistes, restos radiculares y cuerpos extraños intrasinusales, pero también es una imagen en dos dimensiones ${ }^{12}$. El gold standard es la tomografía computarizada (TC) de cavidades paranasales, que identifica todo tipo de lesiones. En los últimos años ha aumentado el uso del cone beam TC o TVD (tomografía volumétrica digital) que tiene una calidad de imagen similar a la TC, con la ventaja de emitir una menor radiación, pues una TC equivale a 75 cone beam TC, y este último a solo 3 días de exposición solar y a entre 20 y 65 miliSievert, dependiendo del equipo y modalidad utilizados $^{13}$. Dirigidamente se debe buscar engrosamiento mucoso/sinusopatía del seno maxilar, velamientos de los senos, parciales o totales, sobre todo unilaterales (Figura 1). Lesiones periapicales, como hipodensidades que pueden corresponder a abscesos 0 granulomas, pudiendo estar 0 no acompañado de dehiscencia de la cortical, cuerpos extraños intrasinusales, entre otros ${ }^{5,14}$. A pesar de la información disponible, es una patología subdiagnosticada, incluso hasta $45 \%$ en algunos reportes ${ }^{15}$.

Los tratamientos propuestos difieren, desde tratamiento médico aislado con antibióticos y/o tratamiento dental del foco infeccioso (obturaciones, endodoncia, apicectomía, etc.) hasta médicoquirúrgico de SPN o combinados. Sin embargo, la literatura revisada indica que el tratamiento dental y médico quirúrgico de SPN combinado tiene mejores resultados que estos tratamientos por separado ${ }^{16,17}$.

\section{OBJETIVO}

A partir de lo anterior es que se ha establecido como objetivo de este estudio, caracterizar los resultados

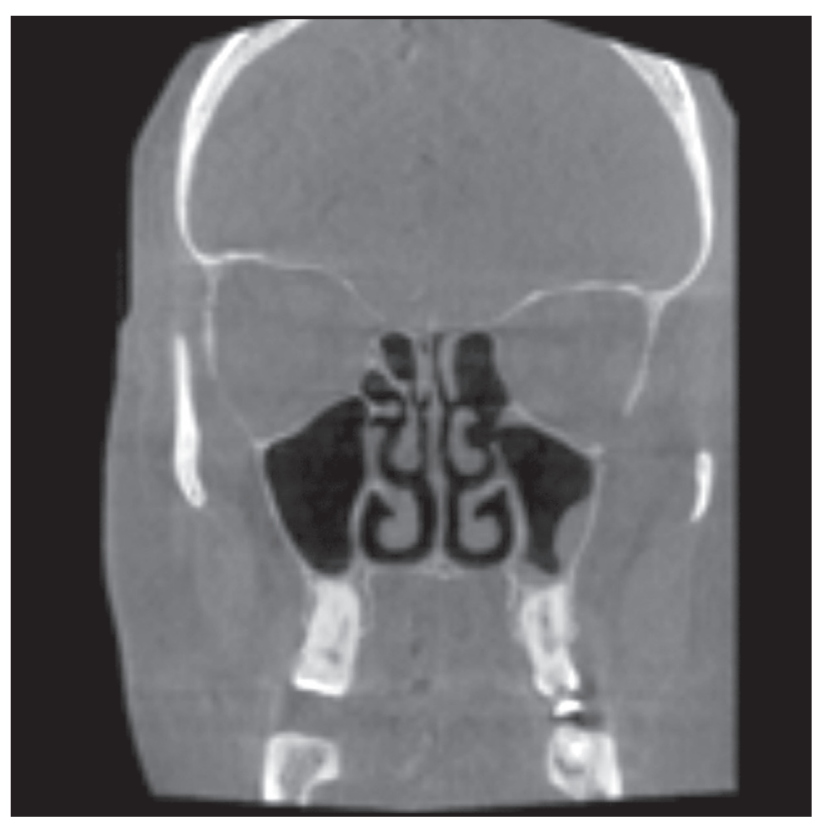

Figura 1. Maxilar izquierdo con engrosamiento mucoso. 
clínicos e imagenológicos de pacientes con RSCO en función del tratamiento recibido, con el fin de poder establecer recomendaciones terapéuticas con respecto al tratamiento otorrinolaringológico y/0 dental. Además de describir la frecuencia de subdiagnóstico de patología dental, el grado de compromiso sinusal radiológico pre y postratamiento, y el grado de resolución de síntomas y signos en función de los tratamientos recibidos.

\section{MATERIAL Y MÉTODO}

Estudio descriptivo, retrospectivo, aprobado por el Comité de Ética de la Universidad de Concepción (UDEC) y financiado por la Vicerrectoría de Investigación y Desarrollo UDEC. Fueron reunidos todos los pacientes con diagnóstico clínico e imagenológico de RSCO entre los años 2013-2017 en un centro de atención médica privado, a los que se invitó a realizar un cone beam TC de control, previo consentimiento informado. De aquellos que aceptaron a participar se accedió a ficha clínica, reuniendo síntomas, imágenes, tratamientos por otorrinolaringólogo y dentales recibidos. Fueron excluidos aquellos con RSC, CEF previa, cáncer, embarazadas, rechazo a la realización del cone beam TC, ausencia de imágenes preoperatorias y menores de 18 años. Los datos fueron tabulados y analizados en Excel. La remisión de síntomas se clasificó en total, parcial o persistente. No se utilizó ningún tipo de cuestionario validado para la evaluación clínica debido a sesgo de memoria de los pacientes dependiendo de su resultado final al que estaría sujeto postratamiento. Se clasificaron las imágenes según grado de compromiso del seno maxilar en: engrosamiento mucoso, velamiento parcial y velamiento total, compromiso del complejo osteomeatal (COM) y grado de extensión a otros SPN. También se describió el tipo de lesión radiológica dental presente y la graduación del compromiso de Ios SPN con el score de Lund-Mackay.

\section{RESULTADOS}

De un total de 64 pacientes, al aplicar los criterios de inclusión y exclusión, permanecieron en el estudio 27. Cincuenta y dos por ciento (14/27) era de sexo femenino, con una edad promedio de 47 años. El 96\% (26/27) era de procedencia urbana y $85 \%$ tenía el antecedente de un procedimiento dental adyacente al seno maxilar comprometido. El tiempo promedio estimado entre la realización de un procedimiento dental y los síntomas de rinosinusitis fue de 9 años. En lo que se refiere al procedimiento dental previo, la mayoría correspondía a endodoncias, seguido de obturaciones y exodoncia. De los pacientes con RSCO 22/27 (81\%) no tenían reportado el foco dental en el informe radiológico médico a pesar de estar presente y visible, lo que fue analizado por nuestro radiólogo máxilo-facial. La herramienta imagenológica inicial utilizada fue cone beam TC en $14 / 27(52 \%)$ y $13 / 27(48 \%)$ TC SPN. La pieza dental más afectada fue el molar, sobre todo el primer y segundo molar.

Clínicamente, la mayoría presentó síntomas inespecíficos, como obstrucción nasal, rinorrea, descarga posterior y opresión facial. Los signos más sugerentes de patología dental fueron, algún síntoma dental en $13 / 27(48 \%)$, hiposmia 9/27 $(33 \%)$ y cacosmia $9 / 27(33 \%)$. El síntoma dental más frecuente fue algia dental presente en $9 / 13$ (69\%) pacientes, seguido de la sensación de pieza dental suelta en 2/13 (15\%). Respecto a los hallazgos radiológicos pretratamiento, la mayoría de los pacientes 24/27 (88\%) presentó compromiso unilateral de SPN, en $7 / 27(25 \%)$ se extendía a otros SPN. Sólo en 4/27 (14\%) comprometía ambos maxilares. Al observar el compromiso radiológico del maxilar, la mayoría 13/27 (48\%) presentó un velamiento parcial, seguido por engrosamiento mucoso y compromiso del complejo osteomeatal ipsilateral. Al aplicar el score de Lund-Mackay, predominó 1 punto y en promedio fue de 2,25 puntos pretratamiento. La lesión dental vista con mayor frecuencia era la hipodensidad periapical en $15 / 27(62 \%)$, seguida de cortical dehiscente $5 / 27(18,5 \%)$, estando ambos presentes en $5 / 27$ $(18,5 \%)$ (Figura 2).

Respecto al tratamiento realizado, 24/27 (89\%) pacientes fueron operados de CEF asociado a un procedimiento dental. De los pacientes sin CEF, (3/27) recibieron tratamiento dental. Uno se trató con endodoncia, el segundo con apicectomía y el tercer caso, no fue especificado, siendo estos tratamientos elegidos por recomendación del odontólo- 


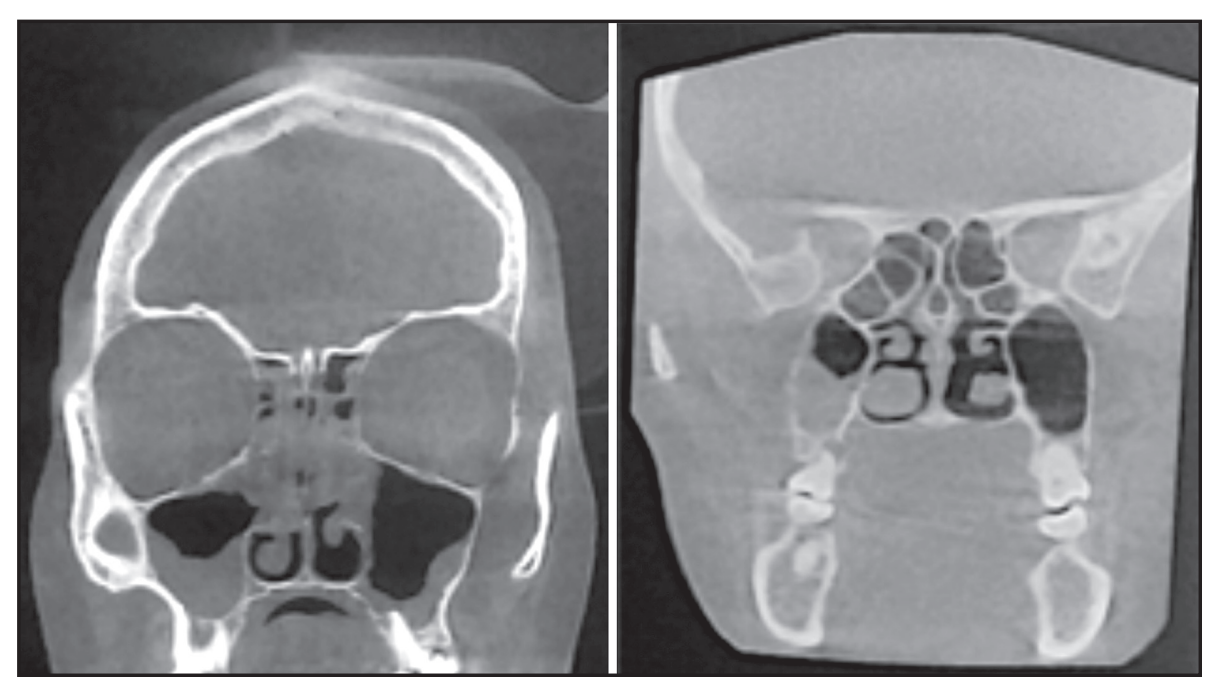

Figura 2: Seno maxilar izquierdo cortical dehiscente y seno maxilar derecho con hipodensidad periapical.

go tratante. En el grupo de tratamientos asociados $(\mathrm{n}=24)$, la CEF se asoció a extracción dental más cierre de fístula en $21 / 24(87,5 \%)$ y solo en $3 / 24$ casos se realizó otro tipo de tratamiento dental. En 20/24 (83\%) casos la CEF y tratamiento dental se realizaron en un tiempo quirúrgico, con la colaboración de un otorrinolaringólogo y un cirujano maxilofacial. De los $4 / 24$ casos con tratamiento diferido, uno tenía una extracción dental previa a la CEF, sin cierre de fístula. En otro caso la extracción del implante causal de la RSCO fue diferida y 2 casos recibieron endodoncias posteriores a la CEF.

\section{Éxito clínico según tratamiento recibido}

De los pacientes que recibieron CEF asociado a un procedimiento dental (24/27), la CEF fue de distinta magnitud. Aquellos en que se realizó solo antrostomía (15/24), en $15 / 15(100 \%)$ tuvo resolución total (RT) de los síntomas. En los que se realizó antrostomía y cirugía de otros SPN (9/24), en $5 / 9(55,5 \%)$ pacientes se observó resolución total de los síntomas, en 3/9 (33\%) resolución parcial (RP) y $1 / 9(11 \%)$ persistencia (PE) de los síntomas. Aquellos que solo recibieron tratamiento dental (3/27) en 1/3 (33\%) tuvo RT de los síntomas, $33 \%$ RP y $33 \%$ PE. En general, de todos los pacientes operados de CEF (24/27), independiente de la extensión de esta misma, la resolución total de los síntomas se observó en 20/24 (83\%), resolución parcial en $3 / 24(12,5 \%)$ y persistencia de los síntomas en 1/24 (4\%). Entre ellos, 3/24 casos recibieron tratamiento diferido ( $\pm 3,5$ meses) logrando RT de los síntomas en 3/3 (Tabla 1).

Tabla 1. Resolución de síntomas según tratamiento recibido

\begin{tabular}{|lccc|}
\hline Grado de resolución & Resolución total & Resolución parcial & Persistencia \\
\hline Tratamiento recibido & & & $0 / 15$ \\
\hline Antrostomía y traitaient dental $(n=15)$ & $15 / 15$ & $3 / 9$ & $1 / 9$ \\
Antrostomía, otros SPN y tratamiento dental $(n=9)$ & $5 / 9$ & $3 / 24$ & $1 / 24$ \\
Todas las CEF más tratamiento dental $(n=24)$ & $20 / 24$ & $1 / 3$ & $1 / 3$ \\
Sólo tratamiento dental $(n=3)$ & $1 / 3$ & $0 / 3$ \\
\hline
\end{tabular}


La extensión de la CEF se realizó según la extensión de la enfermedad. A los que se les realizó solo antrostomía (15/24), todos tenían compromiso maxilar unilateral y en 3 casos llegaba el compromiso al complejo osteomeatal. En ellos, como se dijo anteriormente, 15/15 presentó resolución total de los síntomas. En aquellos que recibieron CEF y tratamiento dental (24/27), en los que se realizó extracción dental con cierre de fístula 21/24, se obtuvo resolución total de los síntomas en $20 / 24$ (83\%), RP en $3 / 24(14 \%)$ y PE de los síntomas en $1 / 24(4,7 \%)$.

\section{Éxito clínico según compromiso radiológico pretratamiento}

La lesión dental radiológica con mayor grado de resolución postratamiento fue la hipodensidad periapical pura en 14/17 pacientes. Cuando se encontraba una cortical dehiscente aislada (5/27) el éxito clínico se observó en $3 / 5$ casos, y al encontrarse ambas lesiones (5/27) se resolvieron totalmente los síntomas en $4 / 5$ pacientes. Al evaluar el resultado clínico según compromiso inflamatorio del seno maxilar, cuando se observó engrosamiento mucoso 10/27, hubo resolución total de los síntomas en $8 / 10(80 \%)$, resolución parcial en $1 / 10(10 \%)$ y persistencia de los síntomas en $1 / 10(10 \%)$ casos. Cuando pretratamiento existía velamiento parcial del seno maxilar (13/27) hubo en 10/13 resolución total de los síntomas, en $2 / 13$ resolución parcial y en 1/13 persistencia de los síntomas. Cuando se encontró velamiento total del seno maxilar (4/13), la respuesta fue resolución total en $3 / 4$ casos, 1/4 se observó resolución parcial de los síntomas. Cuando existió compromiso del complejo osteomeatal (COM) $6 / 27$, la resolución total de los síntomas ocurrió en $5 / 6$ casos y resolución parcial en $1 / 6$ casos. Cuando el compromiso imagenológico comprometía más allá del seno maxilar (7/27), se obtuvo resolución total de síntomas en 5/7 pacientes. Cuando había compromiso maxilar unilateral (23/27) la RT de síntomas se obtuvo en 19/23 pacientes, cuando era maxilar bilateral (4/27), se resolvieron totalmente los síntomas en 2/4 pacientes (Tabla 2).

\section{Éxito clínico según compromiso radiológico postratamiento}

El tiempo promedio del control radiológico fue de 3,2 años después del tratamiento realizado. Todos los pacientes sin hallazgos radiológicos postratamiento (17/27) estaban asintomáticos (17/17). Los pacientes con engrosamiento mucoso (8/27) presentaban resolución total de los síntomas en $3 / 8$ pacientes. Aquellos con velamiento parcial (1/27), todos asintomáticos. El paciente con velamiento total (1/27), resolución parcial de los síntomas. Paciente con ocupación del COM (1/27), resolución parcial 1/1 (Tabla 3).

Los pacientes con Lund-Mackay de 0 puntos 18/27, contaban 17/18 con RT de los síntomas y 1/18 con resolución parcial. Con un puntaje de

Tabla 2. Resolución de síntomas según imagenología pretratamiento

\begin{tabular}{|lccc|}
\hline Grado de resolución & Resolución total & Resolución parcial & Persistencia \\
\hline Compromiso radiológico & & & \\
\hline Engrosamiento mucoso $(n=10)$ & $8 / 10$ & $1 / 10$ & $1 / 10$ \\
Velamiento parcial $(n=13)$ & $10 / 13$ & $2 / 12$ & $1 / 13$ \\
Velamiento total $(n=4)$ & $12 / 13$ & $1 / 13$ & 0 \\
COM $(n=6)$ & $5 / 6$ & $1 / 6$ & 0 \\
Maxilar unilateral $(n=23)$ & $19 / 23$ & $4 / 23$ & $1 / 4$ \\
Maxilar bilateral $(n=4)$ & $2 / 4$ & $1 / 4$ & $1 / 7$ \\
Otros SPN $(n=7)$ & $5 / 7$ & $1 / 7$ & 0 \\
\hline
\end{tabular}

COM: Complejo osteomeatal. Vale para todas las tablas. 
Tabla 3. Resolución de síntomas según imagenología postratamiento

\begin{tabular}{|lccc|}
\hline Grado de resolución & Resolución total & Resolución parcial & Persistencia \\
\hline Compromiso radiológico postratamiento & & & \\
\hline Sin hallazgos patológicos $(n=17)$ & $17 / 17$ & 0 & 0 \\
Engrosamiento mucoso $(n=8)$ & $3 / 8$ & $3 / 8$ & $2 / 8$ \\
Velamiento parcial $(n=1)$ & $1 / 1$ & 0 & 0 \\
Velamiento total $(n=1)$ & 0 & $1 / 1$ & 0 \\
COM $(n=1)$ & 0 & $1 / 1$ & 0 \\
\hline
\end{tabular}

1 (en 7/27 casos, estando asintomáticos 4/7 pacientes. Con 3 puntos $1 / 27$ casos y 5 puntos $1 / 27$, ambos con resolución parcial de los síntomas. En la resolución total de los síntomas (21/27), predominó un Lund-Mackay de 0 puntos en 17/21 pacientes y 1 punto en $4 / 21$. Cuando existía resolución parcial de los síntomas (4/27), se encontró 0 puntos en $1 / 4,1$ punto en $2 / 4$ y 5 puntos en $1 / 4$. Cuando persistieron los síntomas (2/27), 1/2 tenía 1 punto y el otro 3 puntos. Observándose que, a mayor mejoría clínica, existía un cambio radiológico más significativo.

\section{DISCUSIÓN}

En nuestra casuística la principal patología dental identificada fue la endodoncia, diferente a 10 que ocurre en la literatura internacional, donde predominan las exodoncias y los implantes dentales, aumentando en los países desarrollados ${ }^{6,10}$. La mayoría de los pacientes tenía el antecedente de un procedimiento dental previo. La latencia entre el procedimiento dental y el comienzo de los síntomas fue de 9 años promedio, siendo mayor a lo descrito en otras series, con un promedio de 1 a 4 años ${ }^{18}$. Sin embargo, esta información no se reporta en todos los estudios relacionados. Los síntomas prevalentes fueron inespecíficos, comunes para toda rinosinusitis. Los síntomas orientadores de patología dental fueron la cacosmia (9/27) y el dolor dental (9/27) siendo variable su frecuencia en otros reportes y destacando siempre la unilateralidad de los síntomas ${ }^{4,14,19}$.

El método imagenológico utilizado fue la TC y el cone beam TC, sin diferencia en frecuencia, siendo ambos los métodos diagnósticos más sensibles para la detección de patología dental, con varios estudios que avalan el uso de ambos $12,13,20$. En 22/27 (81\%) pacientes con sinusitis crónica, no tenían reportado el foco dental en el informe radiológico médico, a pesar de estar presente y visible, lo que fue analizado por nuestro radiólogo maxilofacial. En todos nuestros pacientes hubo signos radiológicos de patología dental al diagnóstico, sin embargo, en otras publicaciones, están ausentes hasta en $36 \%$ de los casos, con necesidad de un examen clínico dental exhaustivo y la correlación temporal de los síntomas con un procedimiento dental previo ${ }^{14,15}$. En cada TC/cone beam se calculó el score de Lund-Mackay preoperatorio, obteniendo la mayoría entre 1 y 6 puntos, $13 / 25$ casos obtuvieron un punto, siendo el promedio de 2,2521. En el posoperatorio los puntajes fueron de 0 puntos en 18/27 (67\%) y 1 punto en $7 / 27$, siendo el promedio de 0,5 puntos. A pesar de haber sido creado para evaluar sinusitis rinógena, el score de Lund-Mackay demostró diferencias estadísticamente significativas entre el puntaje pre y posoperatorio ( $p<0,05$, Wilcoxon).

El compromiso más allá del maxilar adyacente a la lesión dental estuvo presente en 7/27 (25\%) casos, siendo similar y en algunos casos menores a lo reportado en la literatura ${ }^{14,22}$. La extensión de la CEF dependía del grado de extensión de la enfermedad, siendo operados solo los SPN comprometidos. Al evaluar los resultados radiológicos postratamiento, había normalidad radiológica en $17 / 27$ (59\%) de los casos, sin embargo, en 4/21 (20\%) pacientes asintomáticos no se observaba radiología normal rinosinusal. Así como los pacientes sometidos a CEF y tratamiento dental 
concomitante (24/27), en $8 / 24$ casos se identificaban alteraciones radiológicas, estando el 20/24 asintomáticos. Es frecuente encontrar algún grado de engrosamiento mucoso después de una CEF, Io que se debe más bien al procedimiento quirúrgico y no a la persistencia de la enfermedad, motivo por el que se reportan alteraciones radiológicas después de CEF en pacientes asintomáticos ${ }^{23,24}$. Clínicamente, 21/27 (78\%) pacientes tuvo resolución total de los síntomas, resolución parcial 4/27 $(15 \%)$ y $2 / 27(7 \%)$ persistencia de síntomas. El mayor éxito se obtuvo en aquellos pacientes que recibieron CEF y tratamiento dental, sin diferencias según momento quirúrgico (mismo tiempo o diferido). Además, se pudo observar mejor respuesta en aquellos pacientes con compromiso exclusivo del maxilar y sólo lesión hipodensa periapical. En los casos de los pacientes con resolución parcial y persistencia de los síntomas, hubo factores identificados en relación a la falla de tratamiento, como la bilateralidad, mala dentadura general, falta de combinación de tratamiento dental y CEF o falla técnica (sinequias y oclusión del antro maxilar) (Tabla 4).

En la literatura se describe que el tratamiento médico farmacológico asociado a tratamiento dental tiene un éxito clínico de 52\% aproximadamente. Este porcentaje de éxito cae a $21 \%$ cuando sólo se realiza un tratamiento médico farmacológico y la etiología es un implante dental sobreinfectado ${ }^{16,17}$. Por lo que los antibióticos deben ir siempre asociados a otro tipo de tratamiento. En nuestra casuística la mayoría tuvo previamente (meses) 0 preoperatorio inmediato (semanas) uso de antibió- ticos o solo corticoides inhalados en conjunto con lavados nasales.

\section{CONCLUSIÓN}

Dado los informes radiológicos deficientes, el médico otorrinolaringólogo, debe conocer y sospechar la patología dental, de lo contrario continuará siendo subdiagnosticado.

Como ya se ha demostrado, para la CEF en casos de RSC, el resultado posoperatorio clínico siempre es mejor que el endoscópico y/o radiológico. La CEF combinada con tratamiento dental concomitante, predominantemente exodoncia con cierre de fístula oro-antral tiene un alto grado de éxito. El presente trabajo valida que la opción de un tratamiento otorrinolaringológico y dental concomitante tiene un alto porcentaje de éxito radiológico y clínico en esta patología. Sin embargo, debido al reducido número de pacientes analizados, no pudimos demostrar qué tan extensiva debiera ser la CEF en estos casos y si es que siempre es necesaria. Tampoco fue posible demostrar si siempre es requerida la exodoncia con cierre de FOA o si se pueden seleccionar pacientes para un tratamiento más conservador. Para ello se requieren estudios futuros, randomizados, prospectivos y que consideren la magnitud de la enfermedad en sus aristas, para poder proponer un tratamiento individual a cada caso, basado en la evidencia generada. Además hace falta la creación/validación de escalas en español para medir resultados clínicos, algunos estudios ya se han iniciado ${ }^{25}$.

Tabla 4. Causas de fracaso de tratamiento sinusitis odontogénica

\begin{tabular}{|lcl|}
\hline Fracaso CEF & Resolución parcial & Factor identificado \\
\hline Resolución parcial de síntomas & 4 & Mal estado dental general \\
& & Sólo tratamiento dental \\
& & Falla técnica quirúrgica \\
Pólipos posoperatorios
\end{tabular}




\section{BIBLIOGRAFÍA}

1. Fokkens WJ, Lund VJ, Mullol J, Bachert C, Alobid I, Baroody F, ET AL. European position paper on rhinosinusitis and nasal polyps. Rhinology 2012; 50: 4-305.

2. Hastan D, Fokkens WJ, Bachert C, Newson RB, Bislimovska J, Bockelbrink A, et al. Chronic rhinosinusitis in Europe - An underestimated disease. A GA 2LEN study. Allergy Eur J Allergy Clin Immunol 2011; 66: 1216-23.

3. Mehra P, Murad H. Maxillary sinus disease of odontogenic origin. Otolaryngol Clin N Am 2004; 37: 347-64.

4. Patel NA, Ferguson BJ. Odontogenic sinusitis: an ancient but under-appreciated cause of maxillary sinusitis. Curr Opin Otolaryngol Head Neck Surg 2012; 20: 24-8.

5. Fredriksson M, Öhman A, Flygare L, Tano K. When Maxillary Sinusitis Does Not Heal: Findings on CBCT Scans of the Sinuses With a Particular Focus on the Occurrence of Odontogenic Causes of Maxillary Sinusitis. Laryngoscope Investig Otolaryngol 2017; 2: 442-6.

6. Arias-Irimia 0, Barona-Dorado C, Santos-Marino JA, Martínez-Rodríguez N, Martínez-González JM. Meta-analisis of the etiology of odontogenic maxillary sinusitis. Med Oral Patol Oral Cir Bucal 2010; 15: e70-3.

7. Giovannetti F, Priore P, Raponi I, Valentini V. Endoscopic sinus surgery in sinus-oral pathology. J Craniofac Surg 2014; 25: 991-4.

8. Oberli K, Bornstein MM, von Arx T. Periapical surgery and the maxillary sinus: radiographic parameters for clinical outcome. Oral Surg Oral Med Oral Pathol Oral Radiol Endod 2007; 103: 848-53.

9. Taschieri S, Torretta S, Corbella S, Del Fabbro M, Francetti L, Lolato A, et al. Pathophysiology of sinusitis of odontogenic origin. J Investig Clin Dent 2015; 1-7.

10. LeE KC, LeE SJ. Clinical features and treatments of odontogenic sinusitis. Yonsei Med J2010; 51 : 932-7.

11. Brook I. Microbiology of acute sinusitis of odontogenic origin presenting with periorbital cellulitis in children. Ann Otol Rhinol Laryngol 2007; 116: 386-8.

12. Shahbazian M, Jacobs R. Diagnostic value of $2 D$ and
3D imaging in odontogenic maxillary sinusitis: a review of literature. J Oral Rehabil2012; 39: 294300. doi:10.1111/j.1365-2842.2011.02262.x

13. Malllet M, Bowles WR, McClanahan SL, John MT, Ahmad M. Cone-beam computed tomography evaluation of maxillary sinusitis. J Endodontics 2011; 37: 753-7.

14. Pokorny A, Tataryn R. Clinical and radiologic findings in a case series of maxillary sinusitis of dental origin. Int Forum Allergy Rhinol 2013; 3: 973-9.

15. Wang KL, Nichols BG, Poetker DM, Loehrl TA. Odontogenic sinusitis: a case series studying diagnosis and management. Int Forum Allergy Rhinol 2015; 5: 597-601.

16. Little Re, Long CM, Loehrl TA, Poetker DM. Odontogenic sinusitis: A review of the current literature. Laryngoscope Investig Otolaryngol 2018; 3: 110-4.

17. Legert KG, Zimmerman M, Stierna P. Sinusitis of odontogenic origin: pathophysiological implications of early treatment. Acta Otolaryngol 2004; 124 : 655-63. doi:10.1080/00016480310016866.

18. Troeltzsch M, Pache C, Troeltzsch M, et al. Etiology and clinical characteristics of symptomatic unilateral maxillary sinusitis: A review of 174 cases. J Craniomaxillofac Surg 2015; 43: 1522-9. doi:10.1016/j.jcms.2015.07.021.

19. Longhini AB, Branstetter BF, Ferguson BJ. Unrecognized odontogenic maxillary sinusitis: $A$ cause of endoscopic sinus surgery failure. Am J Rhinol Allergy 2010; 24: 296-300.

20. Kirsch CFE, Bykowski J, Aulino JM, Berger KL, Choudhri AF, Conley dB, et al. ACR Appropriateness Criteria ${ }^{\circledR}$ Sinonasal Disease. J Am Coll Radiol 2017; 14: S550-9.

21. Lund VJ, Kennedy DW. Staging for rhinosinusitis. Otolaryngol Head Neck Surg 1997; 117: S35-S40. doi:10.1016/s0194-5998(97)70005-6.

22. Crovetto-Martínez R, Martin-Arregui FJ, Zabala-López-de-Maturana A, Tudela-Cabello K, Crovetto-de la Torre MA. Frequency of the odontogenic maxillary sinusitis extended to the anterior ethmoid sinus and response to surgical treatment. Med Oral Patol Oral Cir Bucal 2014; 19: e409-13.

23. Stewart MG, Johnson RF. Chronic sinusitis: symptoms versus CT scan findings. Curr Opin Otolaryngol Head Neck Surg 2004; 12: 27-9. 
24. BhattaCharyya N. A comparison of Syntom Scores and Radiographic Staging Systems in Chronic Rhinosinusitis. Am J Rhinol 2005; 19: 175-9.

25. Simuntis R, Vaitkus J, Kubilius $R$, et al. Comparison of Sino-Nasal Outcome Test 22 Symptom Scores in Rhinogenic and Odontogenic Sinusitis. Am J Rhinol Allergy 2019; 33: 44-50. doi:10.1177/1945892418804975. 